OPEN ACCESS

Edited by:

Jean-Paul Deslypere,

Proclin Therapeutic Research Pte

Ltd., Singapore

Reviewed by:

Fathi M. Sherif,

University of Tripoli, Libya Domenico Criscuolo,

Genovax, Italy

*Correspondence:

Shih-Wei Lai

wei@mail.cmuh.org.tw

Specialty section:

This article was submitted to Pharmaceutical Medicine and Outcomes Research,

a section of the journal

Frontiers in Pharmacology

Received: 15 July 2017

Accepted: 23 August 2017 Published: 05 September 2017

Citation:

Liao K-F, Lin C-L and Lai S-W (2017)

Nationwide Case-Control Study Examining the Association between

Tamoxifen Use and Alzheimer's Disease in Aged Women with Breast Cancer in Taiwan.

Front. Pharmacol. 8:612. doi: 10.3389/fphar.2017.00612

\section{Nationwide Case-Control Study Examining the Association between Tamoxifen Use and Alzheimer's Disease in Aged Women with Breast Cancer in Taiwan}

\author{
Kuan-Fu Liao 1,2,3, Cheng-Li Lin ${ }^{4,5}$ and Shih-Wei Lai4,6* \\ ${ }^{1}$ College of Medicine, Tzu Chi University, Hualien, Taiwan, ${ }^{2}$ Department of Internal Medicine, Taichung Tzu Chi General \\ Hospital, Taichung, Taiwan, ${ }^{3}$ Graduate Institute of Integrated Medicine, China Medical University, Taichung, Taiwan, ${ }^{4}$ College \\ of Medicine, China Medical University, Taichung, Taiwan, ${ }^{5}$ Management Office for Health Data, China Medical University \\ Hospital, Taichung, Taiwan, ${ }^{6}$ Department of Family Medicine, China Medical University Hospital, Taichung, Taiwan
}

Background and Objectives: Little is known about the association between tamoxifen use and Alzheimer's disease in women with breast cancer. The study aimed to explore the association between tamoxifen use and Alzheimer's disease in aged women with breast cancer in Taiwan.

Methods: We conducted a retrospective nationwide case-control study using the database of the Taiwan National Health Insurance Program. Totally, 173 female subjects with breast cancer aged $\geq 65$ years with newly diagnosed Alzheimer's disease from 2000 to 2011 were identified as the cases. Additionally, 684 female subjects with breast cancer aged $\geq 65$ years without any type of dementia were selected as the matched controls. The cases and the matched controls were matched with age and comorbidities. Ever use of tamoxifen was defined as subjects who had at least a prescription for tamoxifen before the index date. Never use of tamoxifen was defined as subjects who never had a prescription for tamoxifen before the index date. We used the logistic regression model to calculate the odds ratio (OR) and 95\% confidence interval $(\mathrm{Cl})$ of Alzheimer's disease associated with tamoxifen use.

Results: The OR of Alzheimer's disease was 3.09 for subjects with ever use of tamoxifen (95\% Cl 2.10, 4.55), compared with never use. The OR of Alzheimer's disease was 1.23 for subjects with increasing cumulative duration of tamoxifen use for every 1 year (95\% Cl 1.13, 1.34), compared with never use.

Conclusion: The increased odds of Alzheimer's disease associated with tamoxifen use may be due to the survival effect, not the toxic effect. That is, the longer the tamoxifen use, the longer the patients survive, and the greater the likelihood that she may have a chance to develop Alzheimer's disease.

Keywords: Alzheimer's disease, breast cancer, woman, Taiwan National Health Insurance Program, tamoxifen 


\section{INTRODUCTION}

Tamoxifen has been widely used as an adjuvant therapy for estrogen receptor-positive breast cancer with good efficacy (Davies et al., 2013; Smith, 2014). The side effects of tamoxifen use include vasomotor and gynecologic symptoms, such as hot flashes, night sweats, and vaginal dryness, and other symptoms, such as weight gain, insomnia, and joint aches (Day et al., 1999; Garreau et al., 2006; Smith, 2014). Recent in vitro studies have shown that tamoxifen can protect neuronal cells against oxidative stress-mediated mitochondrial dysfunction (Moreira et al., 2005; Wakade et al., 2008). That is, tamoxifen use may have a potential role for the neurodegenerative disorders (Arevalo et al., 2011, 2012).

Alzheimer's disease is one of the most commonest neurodegenerative disorders. Some evidence has shown that mitochondrial dysfunction may play a role on the pathogenesis of Alzheimer's disease (Sompol et al., 2008; Cadonic et al., 2016). To date, no epidemiological study explores the association between tamoxifen use and Alzheimer's disease in women with breast cancer. Given female breast cancer was the fourth cause of cancer death in Taiwan in 2016 (Ministry of Health and Welfare, 2017a) we conducted a retrospective nationwide case-control study to explore the association between tamoxifen use and Alzheimer's disease in aged women with breast cancer in Taiwan.

\section{MATERIALS AND METHODS}

\section{Data Source}

Taiwan is an independent country with more than 23 million people (Huang and Chang, 2016; Maa and Leu, 2016; Ooi, 2016; Yu et al., 2016; Chen et al., 2017; Lee et al., 2017). We conducted a retrospective nationwide case-control study to analyze the database of the Taiwan National Health Insurance Program. This insurance program began in March 1995 and the enrollment rate was over $99.6 \%$ of 23 million people living in Taiwan in 2015 (Ministry of Health and Welfare, 2017b). The details of the program can be found in previous studies (Lai et al., 2010; Chen et al., 2016; Tsai et al., 2016; Liao et al., 2017a,b). The study was approved by the Research Ethics Committee of China Medical University and Hospital in Taiwan (CMUH-104-REC2-115).

\section{Sampled Subjects}

Totally, 173 Female subjects with breast cancer aged 65 years and older who were newly diagnosed with Alzheimer's disease (ICD9 code 331.0) from 2000 to 2011 were identified as the cases. The date of a subject being diagnosed with Alzheimer's disease was defined as the index date. Additionally, 684 female subjects with breast cancer aged 65 years and older who never had any type of dementia were selected from the same database as the matched controls. The cases and the matched controls were matched with age (every 5-year interval), comorbidities, and the year of index date.

\section{Comorbidities}

Comorbidities which could be potentially related to Alzheimer's disease before the index date were included as follows: alcohol-related disease, cerebrovascular disease, chronic kidney disease, chronic obstructive pulmonary disease, diabetes mellitus, hyperlipidemia, and hypertension. Based on the ICD-9 codes, the diagnosis accuracy of comorbidities has been well-evaluated in previous studies (Lai et al., 2013, 2017a,b; Hung et al., 2015; Shen et al., 2016).

\section{Measurements of Tamoxifen Use and Aromatase Inhibitors Use}

Prescription history of tamoxifen and aromatase inhibitors was included in the study. The measurements of medications use were adapted from previous studies (Lai et al., 2015, 2016; Cheng et al., 2017). Ever use of medications was defined as a subject why had at least a prescription for medications studied before the index date. Never use of medications was defined as a subject who never had a prescription for medications studied before the index date.

\section{Statistical Analysis}

We compared the distributions of the demographic status, tamoxifen use, aromatase inhibitors use, and comorbidities between the cases and the matched controls using the Chi-square test for categorized variables. The $t$-test was used to examine the differences of mean age and mean duration of exposure to tamoxifen between the cases and the matched controls. Only a univariable logistic regression model was used due to no other variable being significantly related to Alzheimer's disease. We further conducted an analysis about the duration-dependent effect of tamoxifen use on the risk of Alzheimer's disease. All analyses were performed by SAS statistical software (version 9.2; SAS Institute, Inc., Cary, NC, United States). The results were considered statistically significant when two-tailed $P$-values were less than 0.05 .

\section{RESULTS}

\section{Characteristics of the Study Population}

Table 1 discloses the characteristics of the study population. We identified 173 cases with newly diagnosed Alzheimer's disease in 2000-2011 and 684 matched controls, with a similar distribution of age. The mean ages (standard deviation) were 80.2 (5.61) years in cases and 80.0 (5.69) years in matched controls, without statistical significance ( $t$-test, $P=0.19)$. The mean durations of exposure of tamoxifen (standard deviation) were 2.55 (2.00) years in cases and 2.28 (1.80) years in matched controls, without statistical significance ( $t$-test, $P=0.15$ ). The cases with Alzheimer's disease were more likely to have a higher proportion of ever use of tamoxifen than the matched controls $(77.5 \%$ vs. $52.6 \%$, Chi-square test, $P<0.001)$. There was no significant difference in the distributions of ever use of aromatase inhibitors and comorbidities between the cases and the matched controls (Chi-square test, $P>0.05$ for all). 
TABLE 1 | Characteristics of cases with Alzheimer's disease and matched controls.

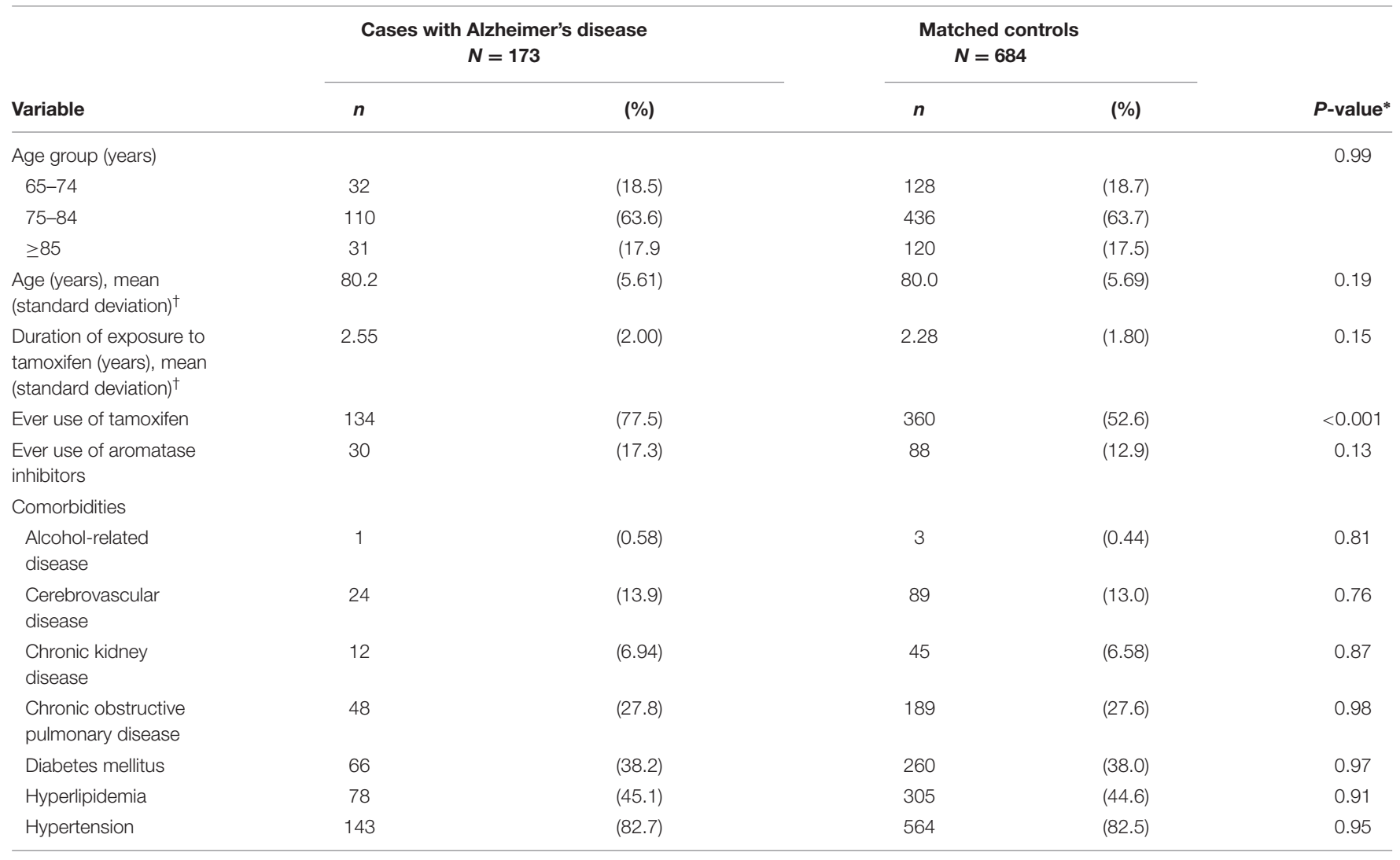

Data are presented as the number of subjects in each group, with percentages given in parentheses, or mean with standard deviation given in parentheses. *Chi-square test, and ${ }^{\dagger} t$-test comparing cases with Alzheimer's disease and matched controls.

TABLE 2 | Odds ratio and 95\% confidence interval of Alzheimer's disease associated with tamoxifen use, aromatase inhibitors use, and comorbidities.

\begin{tabular}{lcc}
\hline Variable & $\mathbf{O R}^{\dagger}$ & $\mathbf{( 9 5 \% \mathbf { C l } )}$ \\
\hline $\begin{array}{l}\text { Age (per 1 year) } \\
\text { Tamoxifen (never use as a reference) }\end{array}$ & 1.02 & $(0.99,1.05)$ \\
$\quad$ Ever use & & \\
Aromatase inhibitors (never use as a reference) & 3.09 & $(2.10,4.55)$ \\
$\quad$ Ever use & 1.42 & $(0.90,2.24)$ \\
Comorbidities (yes vs. no) & & \\
$\quad$ Alcohol-related disease & 1.32 & $(0.14,12.8)$ \\
$\quad$ Cerebrovascular disease & 1.08 & $(0.66,1.75)$ \\
$\quad$ Chronic kidney disease & 1.06 & $(0.55,2.05)$ \\
$\quad$ Chronic obstructive pulmonary disease & 1.01 & $(0.69,1.46)$ \\
$\quad$ Diabetes mellitus & 1.01 & $(0.71,1.42)$ \\
Hyperlipidemia & 1.02 & $(0.73,1.43)$ \\
Hypertension & 1.01 & $(0.65,1.58)$ \\
\hline
\end{tabular}

${ }^{\dagger}$ Because no other variable was significantly related to Alzheimer's disease in a univariable logistic regression model, we did not perform a multivariable logistic regression model.

\section{Risk of Alzheimer's Disease Associated with Tamoxifen Use, Aromatase Inhibitors Use, and Comorbidities}

Table 2 discloses the risk of Alzheimer's disease associated with tamoxifen use, aromatase inhibitors use, and comorbidities. The univariable logistic regression model disclosed that the OR
TABLE 3 | Cumulative duration of tamoxifen use and the risk of Alzheimer's disease.

\begin{tabular}{lccc}
\hline Variable & $\begin{array}{c}\text { Case number } \\
\text { /control number }\end{array}$ & $\mathbf{O R}^{\dagger}$ & $\mathbf{( 9 5 \% ~ C I ) ~}^{+}$ \\
\hline $\begin{array}{l}\text { Never use of tamoxifen } \\
\text { as a reference }\end{array}$ & $39 / 324$ & 1.00 & (Reference) \\
$\begin{array}{l}\text { Cumulative duration of } \\
\text { tamoxifen use (increase } \\
\text { in duration per 1 year) }\end{array}$ & $134 / 360$ & 1.23 & $(1.13,1.34)$ \\
\hline
\end{tabular}

${ }^{\dagger}$ Because no other variable was significantly related to Alzheimer's disease in a univariable logistic regression model, we did not perform a multivariable logistic regression model.

of Alzheimer's disease was 3.09 for subjects with ever use of tamoxifen (95\% CI 2.10, 4.55), compared with never use.

\section{Risk of Alzheimer's Disease Associated with Cumulative Duration of Tamoxifen Use}

Table 3 discloses a sub-analysis on the risk of Alzheimer's disease associated with cumulative duration of tamoxifen use. The OR of Alzheimer's disease was 1.23 for subjects with increasing cumulative duration of tamoxifen use for every 1 year (95\% CI $1.13,1.34)$, compared with never use. 


\section{DISCUSSION}

In this retrospective nationwide case-control study, we noted that tamoxifen use was associated with 3.09-fold increased odds of Alzheimer's disease. We noted that the odds would be increased as the cumulative duration of tamoxifen use was increased (Table 3). It indicates that there could be a duration-dependent effect of tamoxifen use on the risk of Alzheimer's disease. Our findings are contrary to in vitro studies showing that tamoxifen may have a protective role on the neurodegenerative disorders mediated by reducing oxidative stress-related mitochondrial dysfunction (Moreira et al., 2005; Wakade et al., 2008). There seems to be a discrepancy between the epidemiological and in vitro studies. We reviewed the references to explain the discrepancy. The prevalence of Alzheimer's disease is highly associated with increasing age (Qiu et al., 2009; Sosa-Ortiz et al., 2012). That is, the older the patients, the greater the likelihood that he or she would have an Alzheimer's disease. In addition, long-term continuing use of tamoxifen for 10 years could reduce recurrence and mortality of breast cancer, compared with only 5 years of tamoxifen use (Davies et al., 2013; Smith, 2014). That is, long-term of tamoxifen use is associated with improved survival. Thus, patients who use tamoxifen longer may survive longer. They are more likely to develop Alzheimer's disease later. That is, the longer the tamoxifen use, the longer the patients survive, and the greater the likelihood that she may have a chance to develop Alzheimer's disease. Therefore, we think that the increased odds of Alzheimer's disease associated with tamoxifen use may be due to the survival effect, not the toxic effect.

\section{LIMITATION AND STRENGTH}

There are several limitations in this study. First, due to the limitation of a case-control study, a causal relationship between tamoxifen use and Alzheimer's disease cannot be established. Second, our sample size is small. We cannot perform a cohort study. A cohort design does not have the drawback highlighted above for case-control studies. A prospective cohort study with a large sample size is needed for a more detailed analysis of this issue. Third, this study relied on diagnostic codes to establish Alzheimer's disease and other comorbidities, which inevitably introduces some misclassification of cases. In methods section, we mentioned that the cases and the matched controls were matched with comorbidities. It is less likely that misclassification would be more common in tamoxifen users than non-users.

\section{REFERENCES}

Arevalo, M. A., Diz-Chaves, Y., Santos-Galindo, M., Bellini, M. J., and GarciaSegura, L. M. (2012). Selective oestrogen receptor modulators decrease the inflammatory response of glial cells. J. Neuroendocrinol. 24, 183-190. doi: 10.1111/j.1365-2826.2011.02156.x

Arevalo, M. A., Santos-Galindo, M., Lagunas, N., Azcoitia, I., and Garcia-Segura, L. M. (2011). Selective estrogen receptor modulators as brain therapeutic agents. J. Mol. Endocrinol. 46, R1-R9. doi: 10.1677/JME-10-0122
Therefore, the results may not be substantially affected. Fourth, this is a retrospective case-control study. We retrospectively explored whether tamoxifen use has an association with the development of Alzheimer's disease. That is, tamoxifen use precedes the diagnosis of Alzheimer's disease. As a general rule, no anti-Alzheimer's disease drugs can be used before the confirmed diagnosis of Alzheimer's disease is made. Therefore, it is not reasonable to explore any pharmacological interaction between tamoxifen and anti-Alzheimer's disease drugs.

There are several strengths in this study. This is the first nationwide case-control study to explore the association between tamoxifen use and Alzheimer's disease in aged women with breast cancer. The methodology is appropriate. The results are clearly presented. It appears to be informative and influential on its area of research.

\section{CONCLUSION}

The increased odds of Alzheimer's disease associated with tamoxifen use may be due to the survival effect, not the toxic effect. Though tamoxifen use may increase survival, it lets women to be at risk of developing Alzheimer's disease in later life.

\section{AUTHOR CONTRIBUTIONS}

K-FL planned and conducted this study. He participated in the data interpretation, and he revised the article. C-LL conducted the data analysis and revised the article. S-WL planned and conducted this study. He contributed to the conception of the article, initiated the draft of the article, and revised the article.

\section{ACKNOWLEDGMENTS}

This study was supported in part by Taiwan Ministry of Health and Welfare Clinical Trial Center (MOHW106-TDU-B-212113004), China Medical University Hospital, Academia Sinica Taiwan Biobank Stroke Biosignature Project (BM10601010036), Taiwan Clinical Trial Consortium for Stroke (MOST 106-2321-B039-005), Tseng-Lien Lin Foundation, Taichung, Taiwan, Taiwan Brain Disease Foundation, Taipei, Taiwan, and Katsuzo and Kiyo Aoshima Memorial Funds, Japan. These funding agencies did not influence the study design, data collection and analysis, decision to publish, or preparation of the manuscript.

Cadonic, C., Sabbir, M. G., and Albensi, B. C. (2016). Mechanisms of mitochondrial dysfunction in Alzheimer's disease. Mol. Neurobiol. 53, 6078-6090. doi: 10.1007/s12035-015-9515-5

Chen, C. M., Lai, C. H., Wu, H. J., and Wu, L. T. (2017). Genetic characteristic of class 1 integrons in proteus mirabilis isolates from urine samples. Biomedicine 7, 12-17. doi: 10.1051/bmdcn/2017070202

Chen, H. Y., Lin, C. L., Lai, S. W., and Kao, C. H. (2016). Association of selective serotonin reuptake inhibitor use and acute angle-closure glaucoma. J. Clin. Psychiatry 77, e692-e696. doi: 10.4088/JCP.15m10038 
Cheng, K. C., Liao, K. F., Lin, C. L., and Lai, S. W. (2017). Correlation of proton pump inhibitors with pulmonary tuberculosis: a case-control study in Taiwan. Front. Pharmacol. 8:481. doi: 10.3389/fphar.2017.00481

Davies, C., Pan, H., Godwin, J., Gray, R., Arriagada, R., Raina, V., et al. (2013). Long-term effects of continuing adjuvant tamoxifen to 10 years versus stopping at 5 years after diagnosis of oestrogen receptor-positive breast cancer: ATLAS, a randomised trial. Lancet 381, 805-816. doi: 10.1016/S0140-6736(12)61963-1

Day, R., Ganz, P. A., Costantino, J. P., Cronin, W. M., Wickerham, D. L., and Fisher, B. (1999). Health-related quality of life and tamoxifen in breast cancer prevention: a report from the National Surgical Adjuvant Breast and Bowel Project P-1 Study. J. Clin. Oncol. 17, 2659-2669. doi: 10.1200/JCO.1999.17.9. 2659

Garreau, J. R., Delamelena, T., Walts, D., Karamlou, K., and Johnson, N. (2006). Side effects of aromatase inhibitors versus tamoxifen: the patients' perspective. Am. J. Surg. 192, 496-498. doi: 10.1016/j.amjsurg.2006.06.018

Huang, Y. P., and Chang, N. W. (2016). PPARalpha modulates gene expression profiles of mitochondrial energy metabolism in oral tumorigenesis. Biomedicine 6, 17-22. doi: 10.7603/s40681-016-0003-7

Hung, S. C., Liao, K. F., Muo, C. H., Lai, S. W., Chang, C. W., and Hung, H. C. (2015). Hearing loss is associated with risk of Alzheimer's disease: a case-control study in older people. J. Epidemiol. 25, 517-521. doi: 10.2188/jea.JE20140147

Lai, S. W., Lai, H. C., Lin, C. L., and Liao, K. F. (2015). Finasteride use and acute pancreatitis in Taiwan. J. Clin. Pharmacol. 55, 657-660. doi: 10.1002/jcph.462

Lai, S. W., Liao, K. F., Liao, C. C., Muo, C. H., Liu, C. S., and Sung, F. C. (2010). Polypharmacy correlates with increased risk for hip fracture in the elderly: a population-based study. Medicine 89, 295-299. doi: 10.1097/MD. 0b013e3181f15efc

Lai, S. W., Lin, C. L., and Liao, K. F. (2013). No association between gout and Alzheimer's disease: results of a case-control study in older people in Taiwan. Int. J. Geriatr. Psychiatry 28, 1205-1206. doi: 10.1002/gps.3963

Lai, S. W., Lin, C. L., and Liao, K. F. (2016). Use of methimazole and risk of acute pancreatitis: a case-control study in Taiwan. Indian J. Pharmacol. 48, 192-195. doi: 10.4103/0253-7613.178841

Lai, S. W., Lin, C. L., and Liao, K. F. (2017a). Risk of contracting pneumonia among patients with predialysis chronic kidney disease: a population-based cohort study in Taiwan. Biomedicine 7, 42-47. doi: 10.1051/bmdcn/2017070320

Lai, S. W., Lin, C. L., and Liao, K. F. (2017b). Glaucoma may be a nonmemory manifestation of Alzheimer's disease in older people. Int. Psychogeriatr. doi: 10.1017/S1041610217000801 [Epub ahead of print].

Lee, M. R., Lin, C., Lu, C. C., Kuo, S. C., Tsao, J. W., Juan, Y. N., et al. (2017). YC-1 induces G0/G1 phase arrest and mitochondria-dependent apoptosis in cisplatin-resistant human oral cancer CAR cells. Biomedicine 7, 31-42. doi: 10.1051/bmdcn/2017070205

Liao, K. F., Cheng, K. C., Lin, C. L., and Lai, S. W. (2017a). Etodolac and the risk of acute pancreatitis. Biomedicine 7, 25-29. doi: 10.1051/bmdcn/2017070104

Liao, K. F., Huang, P. T., Lin, C. C., Lin, C. L., and Lai, S. W. (2017b). Fluvastatin use and risk of acute pancreatitis: a population-based case-control study in Taiwan. Biomedicine 7, 24-28. doi: 10.1051/bmdcn/2017070317

Maa, M. C., and Leu, T. H. (2016). Src is required for migration, phagocytosis, and interferon beta production in Toll-like receptor-engaged macrophages. Biomedicine 6, 14. doi: 10.7603/s40681-016-0014-4
Ministry of Health and Welfare (2017a). 2016 Statistics of Causes of Death. Available at: http://www.mohw.gov.tw/EN/Ministry/Index.aspx [accessed August 1, 2017].

Ministry of Health and Welfare (2017b). 2016 Taiwan Health and Welfare Report. Available at: http://www.mohw.gov.tw [accessed July 1, 2017].

Moreira, P. I., Custodio, J. B., Oliveira, C. R., and Santos, M. S. (2005). Brain mitochondrial injury induced by oxidative stress-related events is prevented by tamoxifen. Neuropharmacology 48, 435-447. doi: 10.1016/j.neuropharm.2004. 10.012

Ooi, H. (2016). Bedside pleuroscopy in Taiwan: a great vision for criticallyill patients and intensivists. Biomedicine 6, 13. doi: 10.7603/s40681-0160013-5

Qiu, C., Kivipelto, M., and von Strauss, E. (2009). Epidemiology of Alzheimer's disease: occurrence, determinants, and strategies toward intervention. Dialogues Clin. Neurosci. 11, 111-128.

Shen, M. L., Liao, K. F., Tsai, S. M., Lin, C. L., and Lai, S. W. (2016). Herpes zoster correlates with pyogenic liver abscesses in Taiwan. Biomedicine 6, 24-29. doi: 10.7603/s40681-016-0022-4

Smith, G. L. (2014). The long and short of tamoxifen therapy: a review of the ATLAS Trial. J. Adv. Pract. Oncol. 5, 57-60.

Sompol, P., Ittarat, W., Tangpong, J., Chen, Y., Doubinskaia, I., Batinic-Haberle, I., et al. (2008). A neuronal model of Alzheimer's disease: an insight into the mechanisms of oxidative stress-mediated mitochondrial injury. Neuroscience 153, 120-130. doi: 10.1016/j.neuroscience.2008.01.044

Sosa-Ortiz, A. L., Acosta-Castillo, I., and Prince, M. J. (2012). Epidemiology of dementias and Alzheimer's disease. Arch. Med. Res. 43, 600-608. doi: 10.1016/j. arcmed.2012.11.003

Tsai, T. Y., Lin, C. C., Peng, C. Y., Huang, W. H., Su, W. P., Lai, S. W., et al. (2016). The association between biliary tract inflammation and risk of digestive system cancers: a population-based cohort study. Medicine 95:e4427. doi: 10.1097/MD. 0000000000004427

Wakade, C., Khan, M. M., De Sevilla, L. M., Zhang, Q. G., Mahesh, V. B., and Brann, D. W. (2008). Tamoxifen neuroprotection in cerebral ischemia involves attenuation of kinase activation and superoxide production and potentiation of mitochondrial superoxide dismutase. Endocrinology 149, 367-379. doi: 10.1210/en.2007-0899

Yu, C. C., Chien, C. T., and Chang, T. C. (2016). M2 macrophage polarization modulates epithelial-mesenchymal transition in cisplatin-induced tubulointerstitial fibrosis. Biomedicine 6, 29-34. doi: 10.7603/s40681-0160005-5

Conflict of Interest Statement: The authors declare that the research was conducted in the absence of any commercial or financial relationships that could be construed as a potential conflict of interest.

Copyright (c) 2017 Liao, Lin and Lai. This is an open-access article distributed under the terms of the Creative Commons Attribution License (CC BY). The use, distribution or reproduction in other forums is permitted, provided the original author(s) or licensor are credited and that the original publication in this journal is cited, in accordance with accepted academic practice. No use, distribution or reproduction is permitted which does not comply with these terms. 